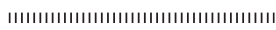

Review

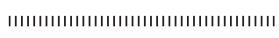

(Special Topic)

\title{
Metabolomics: Methodologies and applications in the environmental sciences
}

\author{
Ching Yu Lin, ${ }^{*}$ Mark R. Viant ${ }^{\dagger}$ and Ronald S. TJEeRdema \\ Department of Environmental Toxicology, University of California, Davis, CA 95616-8588, U.S.A. \\ $\dagger$ School of Biosciences, The University of Birmingham, Edgbaston, Birmingham, B15 2TT, U.K.
}

(Received June 6, 2006)

\begin{abstract}
Environmental metabolomics is an emerging approach for examining metabolic fingerprints, or profiles, in biological systems exposed to environmental stress. In conjunction with other "omics" techniques, such as genomics, transcriptomics, and proteomics, it has been used to study the biochemical impacts of xenobiotics and disease. The approach analyzes changes in the concentrations of metabolites, which are the precursors and products of enzymatic activity, and then attempts to associate these changes with biological function and/or regulation. Environmental scientists have recently applied such techniques to suggest biomarkers for the risk assessment of chemicals and for diagnosing diseases in wild animals. Furthermore, this approach can in principle allow scientists to better understand the underlying mechanisms of action of toxic compounds in the environment. In this review the methodologies used in metabolomics are briefly discussed along with several examples from the environmental sciences, including biomarker development and risk assessment of toxicant exposure, metabolic responses to environmental stressors, and disease diagnosis and monitoring. (C) Pesticide Science Society of Japan
\end{abstract}

Keywords: metabolomics, environment, toxicity, risk assessment, disease, wildlife.

\section{Introduction}

Metabolomics comprises the measurement of low molecular weight endogenous metabolites and can provide an overview of the metabolic status of a biological system. It can examine the physiological condition of a cell or organism and associate the metabolic changes to genetic or environmental modulation. Similar to the fields of genomics and proteomics, metabolomics can examine biological systems at several levels, including cellular, tissue, organ, or even whole organism in response to environmental stressors. ${ }^{1-3)}$ The advantage of metabolomics is that it provides the most functional measure of cellular status and in principle can help to describe an organism's phenotype. The metabolic "fingerprints" of an organism might be altered by genetic or environmental changes, which metabolomics records and then attempts to associate with biological functions. This approach is widely applied in the field of basic biology, ${ }^{4)}$ clinical pharmacology and toxicol-

\footnotetext{
* To whom correspondence should be addressed.

E-mail: clin@ucdavis.edu

(C) Pesticide Science Society of Japan
}

ogy, ${ }^{5-7)}$ and nutrition. ${ }^{8)}$ Environmental scientists have also successfully applied metabolomics to examine toxic impacts or environmental stress on animals, ${ }^{9-11)}$ and to further develop biomarkers to diagnose diseases in wild animals. ${ }^{1,12)}$ It also demonstrates its importance in conjunction with other applications, like proteomics ${ }^{1)}$ and systems biology. ${ }^{13}$

"Omics" can provide answers to questions surrounding biological effects that are caused by exposure to environmental stressors. Furthermore, the approach can assist biologists in their understanding of toxicological mechanisms of action. It is well known that the measurement of genes, mRNA levels, and protein levels cannot be used to directly determine cellular functions. One gene can direct the production of various proteins. Moreover, various post-translational modifications can lead proteins to different locations and functions. ${ }^{14)}$ The chemical environment, such as $\mathrm{pH}$ and redox status, may also affect protein function. Therefore, researchers studying genes or proteins may still require information on small molecular weight metabolites to understand a given biological response since metabolites are crucial to cellular regulatory processes.

Traditional approaches for studying the effects of environmental toxicants or monitoring organismal health in the envi- 
ronment typically include endpoints such as mortality, tumor incidence, reproductive dysfunction, or behavioral effects. Morphological studies can further elucidate these responses in specific cells or tissues by detecting cellular necrosis, but this approach lacks information on the mechanisms of action that leads to the observed endpoint. Without such information, the influence of other environmental factors on organisms will be difficult to predict. The metabolomic approach to environmental research can provide biochemical insight into the mechanisms of stress, increasing both our understanding and the likelihood of detecting toxicants or diseases. Moreover, this approach can generate information from in vivo samples to expand our understanding of the responsible toxicological or pathological mechanisms. ${ }^{15)}$

The metabolomic approach focuses on those metabolites whose levels are altered after stress. It tries to avoid biases against the observation of certain classes of metabolites, based on chemical structure or abundance in biological systems. ${ }^{16)}$ Therefore, the procedures involved in data acquisition and data mining/interrogation require careful consideration as they relate to the interpretation of results. This report discusses analytical methods applied in those procedures and provides several examples of recent applications of metabolomics in environmental research.

\section{Analytical techniques in metabolomics}

Metabolic fingerprints that provide both well-resolved peaks and unambiguous assignments may not be necessary in a fingerprinting-based comparison for diagnosis or product control purposes. ${ }^{16)}$ However, in order to gain insight into biological mechanisms, to model biological processes, and to determine robust biomarkers of toxicity and disease, it is becoming increasingly important to unambiguously identify and quantify metabolites. An increasing number of published methods apply different analytical techniques to metabolomic analyses, such as high-performance liquid chromatography (HPLC) with UV detection, ${ }^{17}$ ) Fourier transformed infrared (FT-IR) spectroscopy, ${ }^{18,19)}$ mass spectrometry $(\mathrm{MS})^{20)}$ and nuclear magnetic resonance (NMR). ${ }^{10,21,22)} \mathrm{MS}$ and NMR are the techniques utilized most commonly since both are able to detect a wide range of metabolites with relatively high specificity and reproducibility. Current MS and NMR techniques in metabolomics have been discussed in some depth ${ }^{23-25)}$ and so our discussion will be limited to only pertinent details in this paper. Even though both techniques offer reliable observation of metabolites it should be noted that neither approach can detect all metabolites in the metabolome.

\section{MS techniques}

MS is commonly used in metabolomics since it provides a blend of sensitive, rapid, and selective qualitative and (potentially) quantitative analyses with the ability to identify metabolites. $^{23)} \mathrm{MS}$ can be used alone or coupled to a chromatographic technique. With no chromatographic separation, direct injection mass spectrometry (DIMS) comprises the injection (or continuous infusion) of a crude sample extract directly into an electrospray mass spectrometer resulting in one spectrum per sample. The composition of the sample can be classified by the mass spectrum or mass list $(\mathrm{m} / \mathrm{z} v \mathrm{~s}$. detector response). This approach provides a quick screening tool, but is not particularly quantitative. DIMS has been applied successfully to the study of microorganisms. ${ }^{26,27)}$

MS combined with chromatography, including liquid chromatography (LC), gas chromatography (GC), or capillary electrophoresis (CE) is widely applied in metabolomic studies. Since HPLC methods became more robust and reliable in the 1970s, this technique has been used to analyze series of metabolites in biological samples. ${ }^{28)}$ HPLC combined with MS has become a sensitive and robust analytical method in metabolomics, with specific software available for metabolite identification. LC-MS or LC-TOF MS (time-of-flight mass spectrometry) derived metabolic fingerprints, in association with statistical analysis, have been applied in the field of pharmacology and toxicology. ${ }^{29-31)}$ In studies of xenobiotics, a metabolomic approach can provide not only information on their biochemical effects in organisms, but also on their biotransformation pathways. The recent development of ultra performance liquid chromatography (UPLC) has resulted in a greater sensitivity and resolution compared to HPLC. UPLC provides more than doubling of peak capacity, an almost 10fold increase in speed, and a 3 - to 5-fold increase in sensitivity compared to conventional reversed phase HPLC. ${ }^{32)}$ UPLCMS has been applied in metabolomics and has yielded an improvement of metabolic classification and pathway differentiation between mouse strains. ${ }^{32}$ )

GC-MS is also an important analytical method for metabolomics. Researchers have used GC-MS analysis to define the prognosis of human neuroendocrine cancers, ${ }^{2)}$ as well as to classify cancer-related and normal tissues. ${ }^{33)}$ Biomarkers of adrenarche examined by GC-MS ${ }^{34)}$ could also provide information for the diagnosis of steroid-related disorders. The GC-MS approach provides exceptional sensitivity, but sample preparation requires significant effort, as many metabolites require derivatization prior to analysis.

Finally, CE-MS has shown its importance in metabolomics, and has been employed to analyze sugar nucleotides in bacterial metabolomics. ${ }^{35)}$

\section{NMR techniques}

Various NMR techniques have been used to record metabolic fingerprints from biological samples. Nicholson et al. ${ }^{21)}$ have employed ${ }^{1} \mathrm{H}$ NMR in analyzing biofluids in order to diagnose diseases since the mid 1980's. Even though NMR is not as sensitive as MS, it is potentially fully quantitative using a single internal standard, is extremely reproducible, employs a highly non-selective detector (metabolite requires a non-exchangeable $\mathrm{H}$ atom), and is able to analyze liquid and solid samples directly. In addition, NMR can be used to detect the 

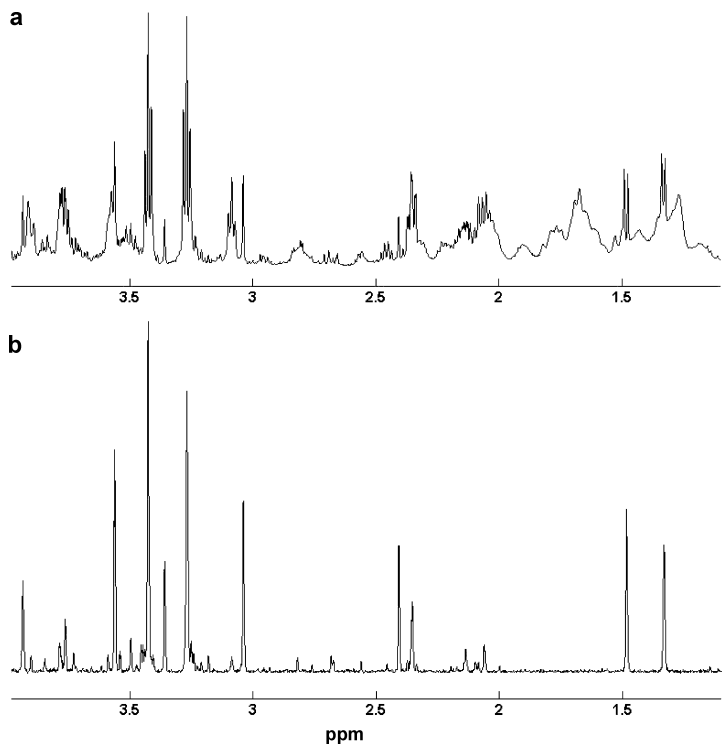

Fig. 1. Representative NMR spectra of a methanol/water $(2 / 1)$ salmon smolt liver extract: a) 1-dimensional (1D) ${ }^{1} \mathrm{H}$ NMR spectrum and b) 1D skyline projection of a 2D J-resolved NMR spectrum.

isotopes of many elements, including ${ }^{13} \mathrm{C},{ }^{31} \mathrm{P}$, and ${ }^{15} \mathrm{~N}$. For instance, biological systems can be exposed to low molecular weight substrates labelled with either ${ }^{13} \mathrm{C}$ or ${ }^{15} \mathrm{~N}$, and the metabolic pathways examined via either ${ }^{13} \mathrm{C}$ or ${ }^{15} \mathrm{~N}$ NMR. ${ }^{36}$ ) This approach would provide not only pathway information, but would also allow for monitoring metabolic changes over time and space.

The presence of macromolecules such as lipoproteins and lipids in samples can cause overlapping resonances and broad peaks in ${ }^{1} \mathrm{H}$ NMR spectra. A few NMR techniques such as spin-echo, ${ }^{37,38)}$ relaxation-editing, ${ }^{39)}$ and ${ }^{1} \mathrm{H},{ }^{1} \mathrm{H}$ J-resolved spectroscopy (Fig. 1) have been shown to enhance metabolite analysis as they provide well-resolved spectral peaks, ${ }^{37,40)}$ which increases the likelihood that a specific metabolite can be unambiguously identified and quantified. NMR can also be used to study intact tissues using magic angle spinning techniques, which reduce the broad and distorted peaks caused by magnetic field inhomogeneity. ${ }^{37,41,42)}$ With this technique, metabolite changes could be localized and further used to study tissue or site-specific mechanisms of toxicity. ${ }^{15,43)}$ The main disadvantage of NMR is its relative insensitivity. However, the use of cryogenically cooled probes and capillary probes has shown considerable promise for increasing sensitivity. ${ }^{44-46)}$

\section{Metabolomics in environmental research}

The application of metabolomics to the environmental sciences is still in its infancy, although the approach offers great potential in several areas. In environmental toxicology, metabolomics can be used to characterize and understand the metabolic or biochemical responses of an organism to toxicant exposure. Such information is of particular value for the risk assessment of chemicals in the environment. In addition, the metabolic responses of organisms to natural environmental stressors, such as heat, cold, or starvation can be characterized using metabolomics, providing insight into organism physiology. Metabolomics can also potentially provide both diagnostic and prognostic indicators for the health of wildlife. The current literature on metabolomics in environmental research may be classified into the following areas: 1) toxicant exposure and risk assessment, 2) responses to environmental stressors, and 3) disease monitoring.

\section{Toxicant exposure and risk assessment}

Metabolomics, in general, does not target specific analytes, but provides an unbiased assessment of the response of organisms to toxic impact as well as an unbiased development of biomarkers for risk assessment. Both terrestrial and aquatic animals have been successfully used to examine the metabolic impacts from environmental toxicant exposures. ${ }^{10,47)}$ Interestingly, almost half of the papers on environmental metabolomic research involve the study of earthworms. Earthworms have been suggested as useful indicators of soil ecosystem health, since they fertilize soil by mixing mineral particles, promote microbial activity, and break down plant organic matter. ${ }^{48)}$ Moreover, earthworms are relatively immobile and are in direct contact with their surrounding environment. Combining NMR-based metabolomics and pattern recognition, several environmental contaminants were examined in earthworm species (Lumbricus rubellus, L. terrestris, and Eisenia andrei) exposed to metal contaminated soil, ${ }^{9)}$ in L. rubellus and E. andrei exposed to copper, ${ }^{49)}$ and in $E$. veneta exposed to 3 -trifluoromethyl-aniline, ${ }^{47)}$ 3-fluoro-4-nitrophenol, ${ }^{50)}$ 4-fluoroaniline, 3,5-difluoroaniline, and 2-fluoro4-methylaniline, ${ }^{51)}$ and 3-trifluoromethylaniline. ${ }^{22)}$ These studies suggest potential biomarkers for ecotoxicological testing, such as increased levels of maltose in L. rubellus after exposure to metal contaminants and decreased levels of acetate and malonate after exposure to 3-fluoro-4-nitrophenol. They also found that these metabolic effects are chemical dependent; 3,5-difluoroaniline and 2-fluoro-4-methylaniline produce similar metabolic effects, but 4-fluoroaniline does not. ${ }^{51)}$

Moreover, there appear to be different metabolic effects among earthworm species. For example, after exposure to metals histidine levels were slightly elevated in L. rubellus, but greatly reduced in L. terrestris. In order to better understand the basal metabolic status and biochemical composition of this potential indicator of soil contamination, metabolic profiles of different species, specific regions or dissected organs of earthworms were studied. ${ }^{22,52,53)}$ Metabolomics allowed differentiation between two morphologically similar earthworm species and highlighted characteristics of specific organs or regions. For example, the metabolic profiles are variable in the coelomic fluids of three Eisenia earthworm species. ${ }^{52)}$ Using earthworms as an indicator organism, these basic studies can provide information for future environmen- 
tal toxicity study and risk assessment, since the basal metabolic variation among species or organs might affect their toxicological response to xenobiotics.

Terrestrial mammals such as the bank vole (Clethrionomys glareolus) have been used to study renal metabolism and the toxicity of arsenic $\left(\mathrm{As}^{3+}\right) .{ }^{15}$ ) Magic angle spinning ${ }^{1} \mathrm{H}$ NMR on the intact renal tissues revealed abnormal lipid profiles and tissue necrosis leading to changes in the water diffusion coefficient in animals exposed to $\mathrm{As}^{3+}$. In contrast, the wood mouse (Apodemus sylvaticus) is less susceptible to $\mathrm{As}^{3+}$ toxicity. ${ }^{15)}$ Such species-specific toxicity is likely due to different basal biochemical profiles. The metabolic profiles of urine, blood plasma, and intact kidney from three wild small mammals: bank vole, wood mouse, white toothed shrew (Crocidura suaveolens), and the laboratory rat (Sprague-Dawley) were compared. ${ }^{54)}$ Bank voles contained the highest concentrations of aromatic amino acids in all tissues and biofluids. In addition, the lipid concentration and composition of the kidney between the wild animals and Sprague-Dawley rat were strikingly different and suggest that results obtained from studies using laboratory animals may not apply to wild species.

There are also numerous studies on the toxic effects of pesticides and other xenobiotics on fish and aquatic invertebrates using NMR-based metabolomics. For example, it has proven to be more sensitive than HPLC-UV for identifying changes in the metabolism of salmon (Oncorhynchus tshawytscha) alevins exposed to dinoseb, diazinon, or esfenvalerate. ${ }^{11)}$ Moreover, the metabolic fingerprints of eyed egg and alevin extracts revealed both dose-dependent and mechanism-specific metabolic responses induced by these pesticides.

Similar studies conducted with Japanese medaka (Oryzias latipes) have demonstrated that the metabolic changes in embryos exposed to dinoseb are correlated with traditional toxic endpoints such as reduced growth and heart rates, abnormal development, and post-exposure mortality. ${ }^{55)}$ Results from ${ }^{1} \mathrm{H}$ NMR metabolomics are also in agreement with those from in vivo ${ }^{31} \mathrm{P}$ NMR and HPLC-UV, in which phosphocreatine utilization for compensation of ATP loss could be used as an indicator of medaka embryotoxicity. ${ }^{56)}$ Medaka embryos were also used to construct developmental metabolic trajectories ${ }^{40)}$ and to understand the metabolic changes during normal embryogenesis as well as trichloroethylene-induced metabolic perturbation during embryogenesis. ${ }^{57)}$ This provided metabolic trajectories not only for the metabolic changes throughout embryo development, but also for the mode of trichloroethylene toxicity during embryogenesis (Fig. 2).

\section{Responses to environmental stressors}

The effects of environmental stressors, such as heat, cold, and starvation on metabolic profiles have been studied in fruit flies, fish, and earthworms. Metabolomics can help to characterize real-time physiological conditions and metabolic perturbations caused by stress. Several metabolites identified

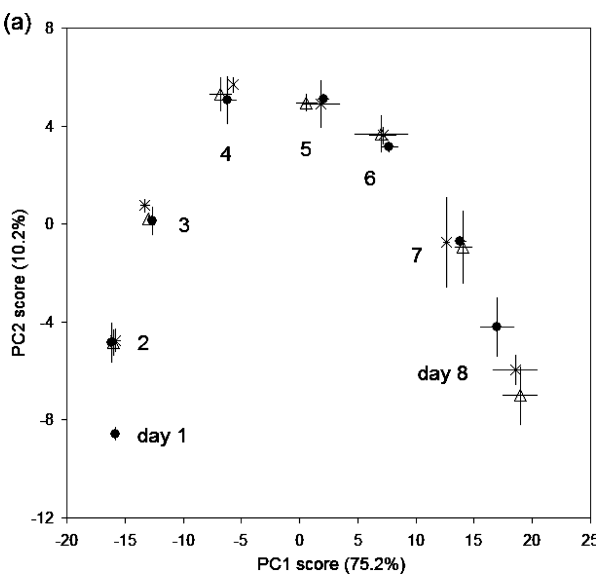

Fig. 2. PCA scores plot of projection of 2D J-resolved spectra of medaka embryos continuously exposed to $0.0(\bullet), 0.88(\triangle)$, and $8.76(\times) \mathrm{mg} / \mathrm{L}$ of trichloroethylene from fertilization (day 1) to immediately prior to hatch (day 8). Trichloroethylene-induced metabolic perturbation is also characterized. (Reprinted with permission from Viant et al., 2005. With kind permission of Springer Science and Business Media.)

were responsive to heat stress in the fruit fly (Drosophila melanogaster) and were correlated to other biochemical and physiological responses. ${ }^{58)}$ For example, the decrease of metabolites (i.e. glucose) involved in energy metabolism is probably caused by the increase in metabolic rate due to elevated temperature. Further, the authors concluded that the temporal development of metabolite concentration and transcriptional levels are strongly correlated. Results from a study of heat stress on steelhead trout (Oncorhynchus mykiss) were used to integrate the description of protein and metabolic responses to thermal stress. The study showed positive correlations between levels of heat-shock induced proteins (hsp72 and hsp89) and decreased levels of energy metabolites (phosphocreatine, ATP, and glycogen). ${ }^{59)}$

Frost stress on earthworms was examined by comparing freeze-tolerant (Dendrobaena octaedra) and freeze-intolerant species. ${ }^{60)}$ After freezing, D. octaedra showed the largest increase of glucose, coupled with a decrease in glycogen. Moreover, relatively lower increases of succinate and lactate in $D$. octaedra suggest anoxic metabolism to be lowest in the freeze-tolerant species. Lenz et al. ${ }^{22)}$ also found distinct metabolic changes (i.e. decreased glucose and increased succinate and lactate) in normal earthworms under cool temperature stress than in tolerant ones. These heat-stress or cold-stress studies provide much needed information for determining enzymatic activity and pathways involved when organisms are subjected to environmental challenge.

Metabolic responses of starvation were also examined in earthworms. Since toxicological tests require earthworms to be starved for a defined period of time, it is important to distinguish the metabolic effects of short-term starvation from toxicant exposure. The metabolic responses of short-term 

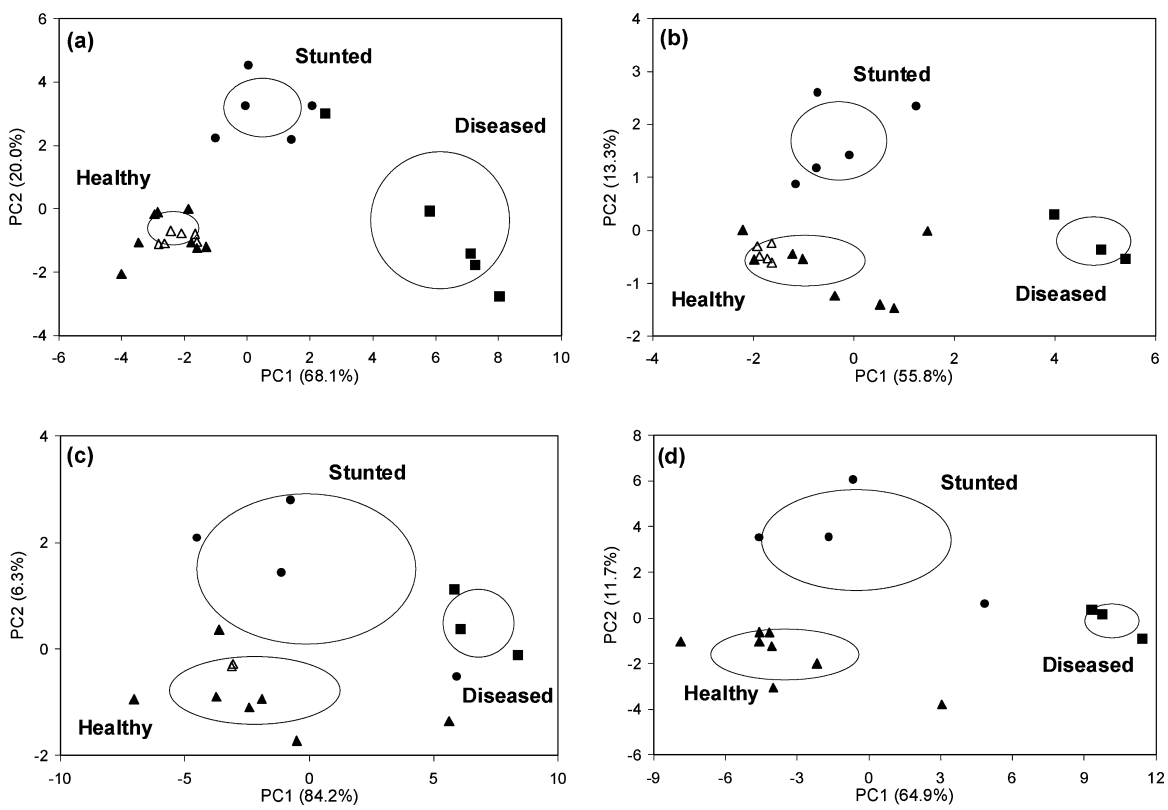

Fig. 3. PCA scores plots of (a) muscle spectra, (b) digestive gland spectra, (c) hemolymph spectra, and (d) a concatenation of all three data sets, clearly separating healthy $(\boldsymbol{\Delta})$, stunted $(\mathbf{O})$, and diseased $(\boldsymbol{\square})$ farm-raised abalone. Replicates obtained from two pooled healthy abalone samples $(\triangle)$ illustrate the consistency of metabolomic analysis. The ellipses represent mean \pm SD (along PC1 and PC2) of each group. (Reprinted with permission from Viant et al., 2003. Copyright 2003 American Chemical Society.)

starvation were determined to be species-dependent. ${ }^{48)}$ While E. veneta showed significant metabolic changes after 6 or 7 days of starvation, L. terrestris showed no consistent metabolic changes after the same period of time. The results suggest that $L$. terrestris might be a better species to use for toxicity testing and monitoring, since the starvation stress does not appear to alter their metabolic profiles.

\section{Disease monitoring}

Metabolomics has also been successfully utilized to characterize diseases in wild aquatic animals, which could be of particular future importance to the aquaculture industry. The metabolic studies highlight the characteristics of liver tumors in flatfish (dab, Limanda limanda) $)^{1)}$ and withering syndrome in red abalone (Haliotis rufescens) ${ }^{10,12)}$ versus corresponding healthy animals (Fig. 3). Stentiford et al. ${ }^{1)}$ have integrated histopathology, proteomics and metabolomics to characterize flatfish liver tumors; several proteins and metabolites were successfully discriminated from fish with liver tumors, and could potentially be utilized in the future as biomarkers to diagnose disease.

Metabolomics can also help to determine the environmental factors leading to diseases such as withering syndrome in California red abalone, ${ }^{12)}$ since alone, Rickettsiales-like prokaryote (RLP) infection is not sufficient to cause withering syndrome. Other environmental stressors such as increased water temperature, acting in combination with RLP infection, have been shown to influence disease status. A particular ratio of two metabolites (glucose and homarine) in foot muscle could serve as potential markers for differentiating the disease status of RLP infected animals. ${ }^{12)}$ Furthermore, the histological measurements also support the NMR metabolomics data in this study.

Solanky et $a l{ }^{61)}$ have also demonstrated that Atlantic salmon infected with the bacterium Aeromonas salmonicida produced a distinct response, leading to metabolic changes in the host plasma that could be used to determine the health status of salmon. Further, the host-pathogen relationship was studied using mutant bacterial strains with deleted effector genes. ${ }^{62)}$ The metabolic profiles from parent and mutant strains suggest that in addition to host invasion there is another required step in $A$. salmonicida pathogenesis.

These studies suggest a potential future role for metabolomics to maintain and monitor the health of animals in both laboratory research and in the aquaculture industry. By studying and characterizing diseased animals, hopefully we can maintain the health, welfare and productivity of aquatic animals in the environment.

\section{Summary}

Some of the primary goals of environmental metabolomics are to distinguish the metabolic fingerprints of environmentally stressed animals from healthy controls, and to identify the most critical biochemical differences between these groups. Even though high-throughput bioanalytical and statistical analyses have been developed or are under development to support this approach, many challenges still remain. For instance, the results from many studies do not show a clear sep- 
aration between the metabolic fingerprints of stressed and control animals. Besides the "technical variance" introduced by the sample extraction and data acquisition, considerable metabolic variation can also arise due to differences in the physiologies of the animals under study. Since physiological condition is dynamic, this introduces yet more complexity in the metabolic fingerprint. Factors that have been shown to effect a metabolic fingerprint include strain, gender and diurnal variation, ${ }^{63,64)}$ age, ${ }^{64)}$ and intestinal microflora. ${ }^{65)}$ Some researchers have demonstrated that metabolic variation can also be due to animal cages, ${ }^{66)}$ or types of drug carrier vehicles. ${ }^{67)}$ Clearly a well designed experiment that attempts to minimize background metabolic variation is extremely important.

If the experimental results show a separation of the metabolic fingerprints of the experimental groups, it is then important to attempt to identify and quantify the metabolic differences. However, the bioanalytical technique used will not have detected all the metabolites in the samples which change concentration either directly or indirectly. Therefore, like other "omics" techniques, it should not be assumed that all or even the most important changes can be identified.

Examining metabolic changes in biological samples provides a useful tool that can assist in the understanding of low molecular weight molecules that regulate organism processes. Although it has some limitations, metabolomics has great potential for facilitating a better understanding of the pathways involved in biological systems in order to predict potential risk factors for wildlife, and to mitigate the effects of exposure to toxicants and disease development in the environment. Metabolomics combined with other "omics" techniques may one day allow researchers to characterize all biochemical interactions that occur within a living system under environmentally stressful conditions.

\section{Acknowledgments}

This project was supported in part by the California Department of Fish and Game's Oil Spill Response Trust Fund through the Oiled Wildlife Care Network at the Wildlife Health Center, School of Veterinary Medicine, University of California, Davis. MR Viant thanks the Natural Environment Research Council, UK for an Advanced Fellowship in Metabolomics (NER/J/S/2002/00618). We thank PA Kuzmicky for editing the manuscript.

\section{References}

1) G. D. Stentiford, M. R. Viant, D. G. Ward, P. J. Johnson, A. Martin, W. Wenbin, H. J. Cooper, B. P. Lyons and S. W. Feist: OMICS 9, 281-299 (2005).

2) J. E. Ippolito, J. Xu, S. Jain, K. Moulder, S. Mennerick, J. R. Crowley, R. R. Townsend and J. I. Gordon: Proc. Natl. Acad. Sci. USA 28, 9901-9906 (2005).

3) E. G. Stanley, N. J. Bailey, M. E. Bollard, J. N. Haselden, C. J. Waterfield, E. Holmes and J. K. Nicholson: Anal. Biochem. 15, 195-202 (2005).

4) S. G. Villas-Boas, S. Noel, G. A. Lane, G. Attwood and A.
Cookson: Anal. Biochem. 349, 297-305 (2006).

5) M. S. Sabatine, E. Liu, D. A. Morrow, E. Heller, R. McCarroll, R. Wiegand, G. F. Berriz, F. P. Roth and R. E. Gerszten: Circulation 112, 3868-3875 (2005).

6) J. Azmi, J. L. Griffin, R. F. Shore, E. Holmes and J. K. Nicholson: Xenobiotica 35, 839-852 (2005).

7) M. E. Bollard, H. C. Keun, O. Beckonert, T. M. Ebbels, H. Antti, A. W. Nicholls, J. P. Shockcor, G. H. Cantor, G. Steven, J. C. Lindon, E. Holmes and J. K. Nicholson: Toxicol. Appl. Pharmacol. 204, 135-151 (2005).

8) J. K. Drackley, S. S. Donkin and C. K. Reynolds: J. Dairy Sci. 89, 1324-1336 (2006).

9) J. G. Bundy, D. J. Spurgeon, C. Svendsen, P. K. Hankard, J. M. Weeks, D. Osborn, J. C. Lindon and J. K. Nicholson: Ecotoxicology 13, 797-806 (2004).

10) M. R. Viant, E. S. Rosenblum and R. S. Tjeerdema: Environ. Sci. Technol. 37, 4982-4989 (2003).

11) M. R. Viant, C. A. Pincetich and R. S. Tjeerdema: Aquat. Toxicol. 77, 359-371 (2006).

12) E. S. Rosenblum, M. R. Viant, B. M. Braid, J. D. Moore, C. S. Friedman and R. S. Tjeerdema: Metabolomics 1, 199-209 (2005).

13) J. K. Nicholson and I. D. Wilson: Nat. Rev. Drug Discov. 2 , 668-676 (2003).

14) S. J. Kwon, E. Y. Choi, Y. J. Choi, J. H. Ahn and O. K. Park: J. Exp. Bot. 57, 1547-1551 (2006).

15) J. L. Griffin, L. Walker and R. F. Shore: Xenobiotica 31, 377-385 (2001).

16) O. Fiehn: Plant Mol. Biol. 48, 155-171 (2002).

17) M. Defernez, Y. M. Gunning, A. J. Parr, L. V. Shepherd, H. V. Davies and I. J. Colquhoun: J. Agric. Food Chem. 52, 6075-6085 (2004).

18) E. Gidman, R. Goodacre, B. Emmett, A. R. Smith and D. Gwynn-Jones: Phytochemistry 63, 705-710 (2003).

19) G. G. Harrigan, R. H. LaPlante, G. N. Cosma, G. Cockerell, R. Goodacre, J. F. Maddox, J. P. Luyendyk, P. E. Ganey and R. A. Roth: Toxicol. Lett. 146, 197-205 (2004).

$20)$ I. D. Wilson, R. Plumb, J. Granger, H. Major, R. Williams and E. M. Lenz: J. Chromatogr. B 817, 67-76 (2005).

21) J. K. Nicholson, J. A. Timbrell and P. J. Sadler: Mol. Pharmacol. 27, 644-651 (1985).

22) E. M. Lenz, J. M. Weeks, J. C. Lindon, D. Osborn and J. K. Nicholson: Metabolomics 1, 123-136 (2005).

23) W. B. Dunn and D. I. Ellis: Trend Anal. Chem. 24, 285-294 (2005).

24) I. Pelczer: Curr. Opin. Drug Discov. Devel. 8, 127-133 (2005).

25) S. G. Villas-Boas, S. Mas, M. Akesson, J. Smedsgaard and J. Nielsen: Mass Spectrom. Rev. 24, 613-646 (2005).

26) J. I. Castrillo, A. Hayes, S. Mohammed, S. J. Gaskell and S. G. Oliver: Phytochemistry 62, 929-937 (2003).

27) J. Allen, H. M. Davey, D. Broadhurst, J. K. Heald, J. J. Rowland, S. G. Oliver and D. B. Kell: Nat. Biotechnol. 21, 692-696 (2003).

28) G. Jones and H. F. DeLuca: J. Lipid Res. 16, 448-453 (1975).

29) R. S. Plumb, C. L. Stumpf, M. V. Gorenstein, J. M. CastroPerez, G. J. Dear, M. Anthony, B. C. Sweatman, S. C. Connor and J. N. Haselden: Rapid Commun. Mass Spectrom. 16, 
1991-1996 (2002).

30) R. S. Plumb, C. L. Stumpf, J. H. Granger, J. Castro-Perez, J. N. Haselden and G. J. Dear: Rapid Commun. Mass Spectrom. 17, 2632-2638 (2003).

31) K. C. Verhoeckx, S. Bijlsma, S. Jespersen, R. Ramaker, E. R. Verheij, R. F. Witkamp, J. van der Greef and R. J. Rodenburg: Int. Immunopharmacol. 4, 1499-1514 (2004).

32) I. D. Wilson, J. K. Nicholson, J. Castro-Perez, J. H. Granger, K. A. Johnson, B. W. Smith and R. S. Plumb: J. Proteome Res. 4, 591-598 (2005).

33) K. R. Kim, H. G. Park, M. J. Paik, H. S. Ryu, K. S. Oh, S. W. Myung and H. M. Liebich: J. Chromatogr. B. Biomed. Sci. Appl. 712, 11-22 (1998).

34) T. Remer, K. R. Boye, M. F. Hartmann and S. A. Wudy: J. Clin. Endocrinol. Metab. 90, 2015-2021 (2005).

35) E. C. Soo, A. J. Aubry, S. M. Logan, P. Guerry, J. F. Kelly, N. M. Young and P. Thibault: Anal. Chem. 76, 619-626 (2004).

36) J. Kikuchi, K. Shinozaki and T. Hirayama: Plant Cell Physiol. 45, 1099-1104 (2004).

37) Y. Wang, M. E. Bollard, H. Keun, H. Antti, O. Beckonert, T. M. Ebbels, J. C. Lindon, E. Holmes, H. Tang and J. K. Nicholson: Anal. Biochem. 323, 26-32 (2003).

38) Q. N. Van, G. N. Chmurny and T. D. Veenstra: Biochem. Biophys. Res. Commun. 301, 952-959 (2003).

39) H. Tang, Y. Wang, J. K. Nicholson and J. C. Lindon: Anal. Biochem. 325, 260-272 (2004).

40) M. R. Viant: Biochem. Biophys. Res. Commun. 310, 943-948 (2003).

41) S. D. Huhn, C. M. Szabo, J. H. Gass and A. E. Manzi: Anal. Bioanal. Chem. 378, 1511-1519 (2004).

42) H. C. Keun: Pharmacol. Ther. 109, 92-106 (2006).

43) S. Garrod, E. Humpher, S. C. Connor, J. C. Connelly, M. Spraul, J. K. Nicholson and E. Holmes: Magn. Reson. Med. 45, 781-790 (2001).

44) H. C. Keun, O. Beckonert, J. L. Griffin, C. Richter, D. Moskau, J. C. Lindon and J. K. Nicholson: Anal. Chem. 74, 4588-4593 (2002).

45) J. L. Griffin, A. W. Nicholls, H. C. Keun, R. J. Mortishire-Smith, J. K. Nicholson and T. Kuehn: Analyst 127, 582-584 (2002).

46) J. L. Griffin, H. Keun, C. Richter, D. Moskau, C. Rae and J. K. Nicholson: Neurochem. Int. 42, 93-99 (2003).

47) M. A. Warne, E. M. Lenz, D. Osborn, J. M. Weeks and J. K. Nicholson: Biomarkers 5, 56-72 (2000).

48) M. A. Warne, E. M. Lenz, D. Osborn, J. M. Weeks and J. K. Nicholson: Soil Biol. Biochem. 33, 1171-1180 (2001).

49) J. O. T. Gibb, C. Svendsen, J. M. Weeks and J. K. Nicholson:
Biomarkers 2, 295-302 (1997).

50) J. G. Bundy, D. Osborn, J. M. Weeks, J. C. Lindon and J. K. Nicholson: FEBS Lett. 500, 31-35 (2001).

51) J. G. Bundy, E. M. Lenz, N. J. Bailey, C. L. Gavaghan, C. Svendsen, D. Spurgeon, P. K. Hankard, D. Osborn, J. A. Weeks and S. A. Trauger: Environ. Toxicol. Chem. 21, 1966-1972 (2002).

52) J. G. Bundy, D. J. Spurgeon, C. Svendsen, P. K. Hankard, D. Osborn, J. C. Lindon and J. K. Nicholson: FEBS Lett. 521, $115-120$ (2002)

53) J. O. T. Gibb, E. Holmes, J. K. Nicholson and J. M. Weeks: Comp. Biochem. Physiol. 118B, 587-598 (1997).

54) J. L. Griffin, L. A. Walker, S. Garrod, E. Holmes, R. F. Shore and J. K. Nicholson: Comp. Biochem. Physiol. 127B, 357-367 (2000)

55) M. R. Viant, C. A. Pincetich, D. E. Hinton and R. S. Tjeerdema: Aquat. Toxicol. 76, 329-342 (2006).

56) C. A. Pincetich, M. R. Viant, D. E. Hinton and R. S. Tjeerdema: Comp. Biochem. Physiol. C. Toxicol. Pharmacol. 140, 103-113 (2005).

57) M. R. Viant, J. G. Bundy, C. A. Pincetich, J. S. de Ropp and R. S. Tjeerdema: Metabolomics 1, 149-158 (2005).

58) A. Malmendal, J. Overgaard, J. G. Bundy, J. G. Sorensen, N. C. Nielsen, V. Loeschcke and M. Holmstrup: Am. J. Physiol. Regul. Integr. Comp. Physiol. In press (2006).

59) M. R. Viant, I. Werner, E. S. Rosenblum, A. S. Gantner and R. S. Tjeerdema: Fish Physiol. Biochem. 29, 159-171 (2003).

60) J. G. Bundy, H. Ramlov and M. Holmstrup: Cryoletters 24, 347-358 (2003).

61) K. S. Solanky, I. W. Burton, S. L. MacKinnon, J. A. Walter and A. Dacanay: Dis. Aquat. Organ. 65, 107-114 (2005).

62) A. Dacanay, L. Knickle, K. S. Solanky, J. M. Boyd, J. A. Walter, L. L. Brown, S. C. Johnson and M. Reith: Microbiology 152, 1847-1856 (2006).

63) R. Plumb, J. Granger, C. Stumpf, I. D. Wilson, J. A. Evans and E. M. Lenz: Analyst 128, 819-823 (2003).

64) R. S. Plumb, J. H. Granger, C. L. Stumpf, K. A. Johnson, B. W. Smith, S. Gaulitz, I. D. Wilson and J. Castro-Perez: Analyst 130, 844-849 (2005).

65) R. E. Williams, H. W. Eyton-Jones, M. J. Farnworth, R. Gallagher and W. M. Provan: Xenobiotica 32, 783-794 (2002).

66) D. G. Robertson, M. D. Reily, J. C. Lindon, D. Holmes and J. K. Nicholson: Compr. Toxicol. 14, 583-610 (2002).

67) B. M. Beckwith-Hall, E. Holmes, J. C. Lindon, J. Gounarides, A. Vickers, M. Shapiro and J. K. Nicholson: Chem. Res. Toxicol. 15, 1136-1141 (2002). 